\title{
Septum transversum diaphragmatic hernia in an adult
}

\author{
Massimiliano Paci, MD, a Salvatore de Franco, MD, ${ }^{a}$ Edoardo Della Valle, MD, ${ }^{b}$ \\ Guglielmo Ferrari, MD, ${ }^{a}$ Valerio Annessi, MD, ${ }^{a}$ Tommaso Ricchetti, MD, ${ }^{a}$ and \\ Giorgio Sgarbi, MD, ${ }^{a}$ Reggio Emilia, Italy
}

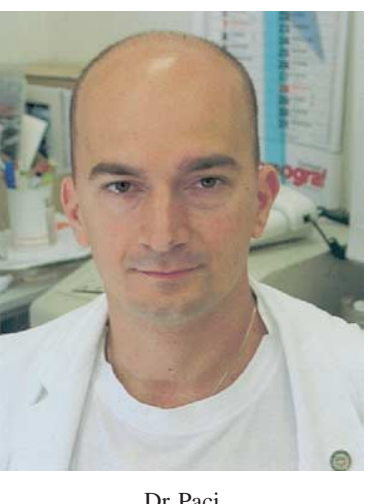

entral diaphragmatic hernia is a rare defect of the coelomic cavity and, in particular, of the septum transversum. An absent or undeveloped pars sternalis of the septum transversum determines the persistence of the pericardial-peritoneal canal, with more or less wide communication between the pericardial and the peritoneal cavities. This malformation can be associated in various ways with other midline congenital defects and Cantrell pentalogy. ${ }^{1}$ No diagnostic case in adults has ever been described in the literature. We thus report an incidentally diagnosed case in an adult that was repaired with laparoscopy.

\section{Clinical Summary}

The patient is a 58-year-old white man whose family history and medical history are unremarkable. During the interview, he reported some recent episodes of melena. The patient was admitted to the department of internal medicine, where laboratory tests revealed severe anemia. Thoracic radiography was performed, revealing a retrosternal, anterobasal, mediastinal air-fluid level. A subsequent thoracoabdominal computed tomographic scan showed an anterior retrosternal diaphragmatic hernia (Figure 1) identified as a Morgagni-Larrey hernia, with herniation in the thoracic cavity of the fundus of the stomach, peritoneal fat, and some intestinal loops (Figure 2). The patient thus underwent a gastroscopy, which showed chronic gastritis. Results of echocardiography and electrocardiography were negative. Respiratory function tests revealed mild obstructive airflow and a moderate restrictive ventilatory disorder. The patient underwent a laparoscopy to repair the hernia.

At the operation, the patient was given general anesthesia with a single-lumen orotracheal tube. The camera was inserted through a 10-mm port (Endopath $10 \mathrm{~mm}$; Ethicon Endo-Surgery, Inc, Cincinnati, Ohio) placed in the left pararectal site; 2 additional working ports were placed for the insertion of dissection instruments in the left superumbilical and subcostal sites. Exploration of the peritoneal cavity showed a central diaphragmatic hernia of 19 $\times 15 \mathrm{~cm}$, with wide communication between the pericardial and

From the Division of Thoracic Surgery a and the 1st Department of General Surgery, Laparoscopic Surgery Unit, , Arcispedale Santa Maria Nuova, Reggio Emilia, Italy.

Received for publication May 11, 2004; revisions received May 27, 2004; accepted for publication June 2, 2004.

Address for reprints: Massimiliano Paci, MD, Division of Thoracic Surgery, Viale Risorgimento 80, 42100 Reggio Emilia, Italy (E-mail: paci.massimiliano@asmn.re.it).

J Thorac Cardiovasc Surg 2005;129:444-5

$0022-5223 / \$ 30.00$

Copyright $\odot 2005$ by The American Association for Thoracic Surgery

doi:10.1016/j.jtcvs.2004.06.015

peritoneal cavities (Figure 3). The diaphragmatic defect was closed with a polytetrafluoroethylene DualMesh patch (Gore-Tex patch; W. L. Gore \& Associates, Inc, Flagstaff, Ariz) attached to the diaphragm with a double layer of titanium helical fasteners (ProTack 5 mm, AutoSuture; Tyco Healthcare Group LP, Norwalk, Conn). There was no evidence of subcutaneous neck emphysema at the end of the operation, and the chest radiograph showed no pneumomediastinum. The patient had an uneventful postoperative course with the exception of minimal pericardial effusion. Three months later, he is doing well without any radiographic evidence of recurrence.

\section{Discussion}

The diaphragm develops in the embryo during the third week of gestation and is made up of 4 elements: the septum transversum, 2 pleuroperitoneal folds, cervical myotomes, and the dorsal mesentery. Its development is complete between the eighth and tenth weeks of gestation. The congenital defect that determines the congenital diaphragmatic hernias (CDHs) is due to the failed development of the septum transversum, the failed development of the pleuroperitoneal folds or improper or absent migration of the diaphragmatic musculature, or weaknesses in points of embryologic fusion. $\mathrm{CDHs}$ have an incidence of 0.08 to 0.45 cases per 1000 births. The septum transversum diaphragmatic hernia occurs in only $1 \%$ to $6 \%$ of CDHs. ${ }^{2}$ Most patients have clinical symptoms at birth or in infancy, with respiratory stress and cardiac dysfunction. However, a small number might present the first symptoms in adulthood, and these are generally caused by complications of the herniated gut, such as strangulation or perforation.

Repair of the hernia can be performed with either a thoracic or abdominal approach, although the latter is preferred because reducing the herniated organs in their own cavities is a top priority.

In 1761, Morgagni described a case of congenital anterior diaphragmatic hernia, and Bochdalek, in 1848, described a case of congenital posterolateral diaphragmatic hernia. ${ }^{3}$

Cantrell and colleagues, ${ }^{1}$ in 1958 , described a congenital defect characterized by a cleft lower sternum, an anterior diaphragmatic defect caused by failure of the development of the septum transversum, absence of parietal pericardium, omphalocele, and cardiac anomaly, frequently called the tetralogy of Fallot.

The first case of isolated central anterior diaphragmatic hernia from a defect of the septum transversum dates back to $1966 .{ }^{4}$ Since then, other cases, all in infancy and childhood, have been reported. ${ }^{2,5}$ Our case is a CDH of an isolated defect of the septum transversum diagnosed incidentally in a patient undergoing laparoscopy for a suspected Morgagni-Larrey hernia. In fact, the defect 


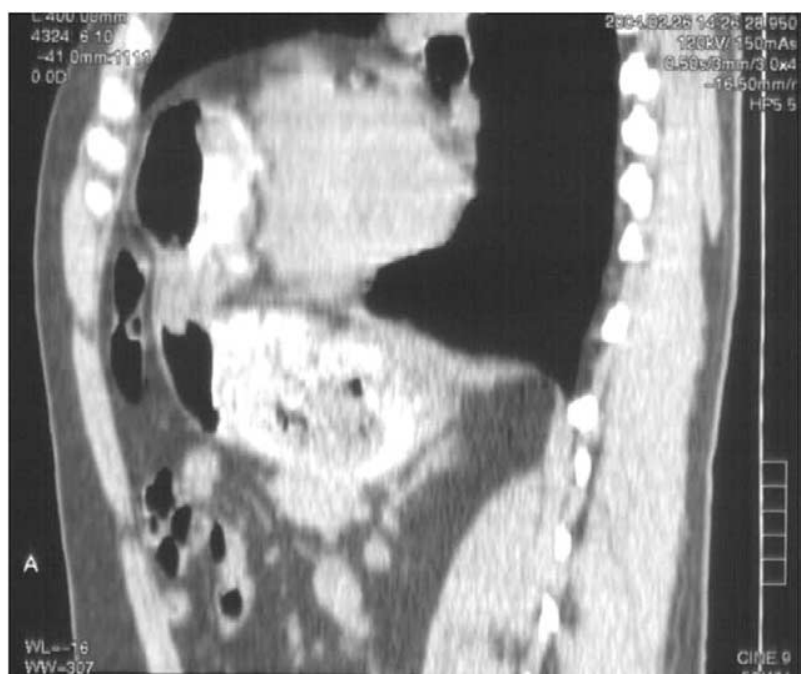

Figure 1. Sagittal reconstruction of thoracomediastinal computed tomogram showing an anterior retrosternal diaphragmatic hernia containing a portion of the stomach and intestinal loops.

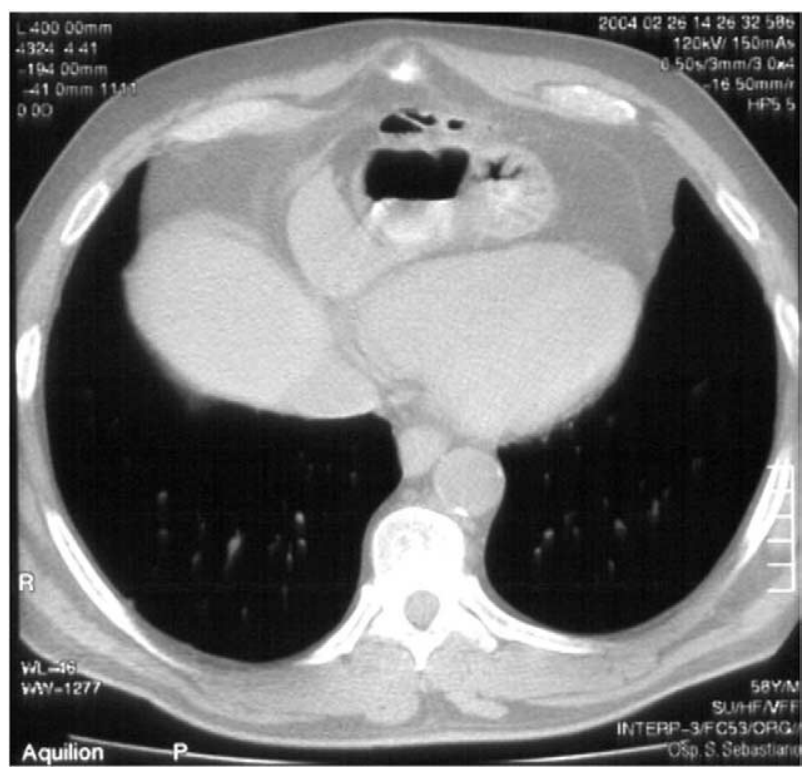

Figure 2. Thoracomediastinal computed tomogram showing herniation in the thoracic cavity of part of the fundus of the stomach highlighted by contrast medium, peritoneal fat, and some loops that do not appear dilated. The heart is pressed in front by the hernia.

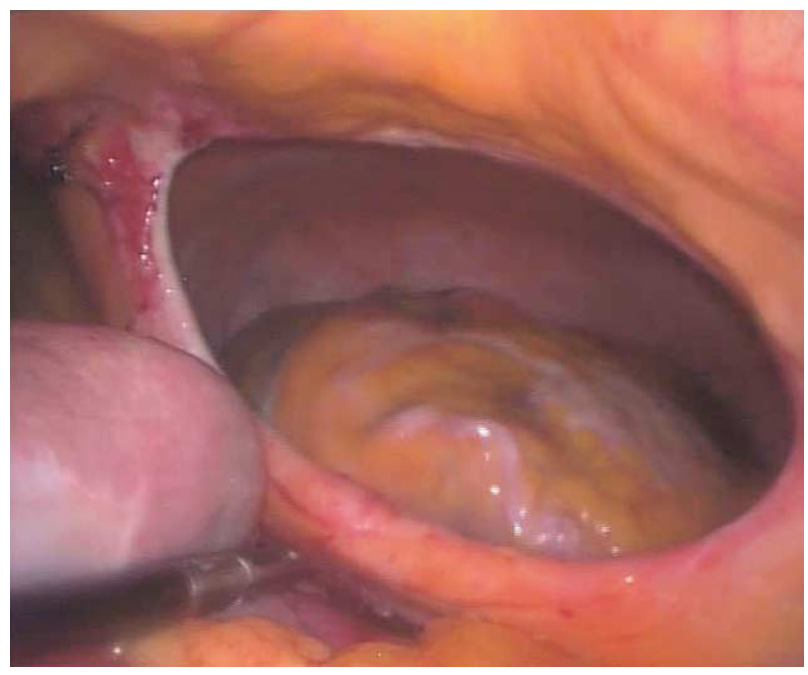

Figure 3. Laparoscopic image of the septum transversum diaphragmatic hernia with widespread pericardial-peritoneal communication. On the left is the left lobe of the liver; at the bottom is the herniated omentum.

was not situated in the sternocostal hiatus or in the parasternal or retrosternal site but rather in the central site with an intact anterior pad and no hernial sac. Repair was carried out successfully by laparoscopy, with no complications.

Therefore, we conclude that the septum transversum diaphragmatic hernia, although extremely uncommon, should be considered in differential diagnosis, even in adult patients with suspected Morgagni hernia, and that it can be safely and effectively repaired by laparoscopy.

\section{References}

1. Cantrell JR, Haller JA, Ravitch MM. A syndrome of congenital defects involving the abdominal wall, sternum, diaphragm, pericardium and heart. Surg Gynecol Obstet. 1958;107:602-14.

2. Clavert JM, De Geeter B, Bientz J, Sauvage P, Buck P. Abdominopericardial hernia as a result of an anomaly of the septum transversum. Apropos of a case in a newborn infant. Chir Pediatr. 1983;24:137-9.

3. Langer JC. Congenital diaphragmatic hernia. Chest Surg Clin North Am. 1998;8:295-314.

4. Pomputius WF, Fisher ER. Intrapericardial fat pads and anomalous development of the septum transversum as a cause of pseudocardiomegaly. Am J Clin Pathol. 1966;46:92-8.

5. Wesselhoeft CW Jr, DeLuca FG. Neonatal septum transversum diaphragmatic defects. Am J Surg. 1984;147:481-5. 